\title{
Traitement des margines par électrocoagulation
}

\author{
N. Sadif, M. Mountadar, F. Hanafi
}

\author{
Unité de chimie analytique et génie de l'environnement du Laboratoire de l'eau et de l'environnement \\ Département de chimie, faculté des Sciences, Université Chouaib Doukkali - BP 20 - 24000 El Jadida, Maroc
}

Pour toute correspondance : n_sadif@yahoo.fr ; mounta_dar@yahoo.fr

\section{Résumé}

Les pays méditerranéens sont très concernés par les problèmes de pollution causés par la production des margines, qui sont les effluents provenant de la production d'huile d'olive. Pour y faire face, les recherches scientifiques se multiplient pour proposer des alternatives au déversement direct de ces effluents dans le milieu naturel.

Dans cette étude, nous nous intéressons au traitement des margines par voie électrochimique. Les paramètres opératoires étudiés sont le $\mathrm{pH}$, le temps d'électrolyse et le courant imposé. Les résultats obtenus ont permis d'obtenir des taux de décoloration pouvant atteindre $95 \%$ pour un $\mathrm{pH}$ égal à 7, un temps d'électrolyse de 15 minutes et un courant imposé égal à 0,5 A. Ces conditions ont permis également des abattements importants de la DCO.

Mots clés : margine, procédés électrochimiques

\section{Introduction}

Les pays méditerranéens sont confrontés à la problématique de l'élimination des margines provenant de la production d'huile d'olive. Ces dernières sont généralement rejetées dans le milieu naturel sans aucun traitement préalable, engendrant ainsi des problèmes graves de pollution pour l'environnement. En effet, ces effluents ont un fort pouvoir polluant dû à une forte charge en demande chimique et biologique en oxygène (DCO et DBO5) $[1 ; 2 ; 3]$ et à la présence de composés phénoliques et aromatiques qui sont très difficilement biodégradables $[4 ; 5 ; 6]$.

De nombreuses études ont été effectuées dans le but de traiter ces effluents en proposant des méthodes économiques de traitement telles que les traitements biologiques $[3 ; 10$; II ; I2], les traitements chimiques par oxydation [3] ou par coagulation floculation $[7 ; 8 ; 9]$.

Notre travail consiste à réduire la matière organique, la coloration et les phénols par électrocoagulation $[13 ; 14 ; 15$; 16]. II s'agit d'une électrolyse d'un effluent coloré qui présente une forte charge en matière polluante, provenant de la région de Marrakech. L'objectif de cette étude est de traiter l'effluent pour le rendre incolore, pour diminuer sa DCO et son contenu en phénols, afin de respecter les normes marocaines en vigueur.

\section{Matériels et méthodes}

Pour effectuer le traitement électrochimique de l'effluent, nous avons utilisé un montage expérimental composé de deux électrodes métalliques espacées de $2 \mathrm{~cm}$ et plongées dans l'effluent. L'aire totale de la surface effective d'électrode est $9 \mathrm{~cm}^{2}$. Le courant imposé est mesuré par un ampèremètre. Dans chaque essai, on traite $100 \mathrm{~mL}$ d'échantillon, dans la cellule électrolytique sous agitation. Le $\mathrm{pH}$ initial des échantillons est ajusté par ajout d'acide sulfurique pour obtenir un milieu acide et par ajout de chaux pour un milieu alcalin. Toutes les mesures sont effectuées après filtration des échantillons traités et les électrodes sont bien polies avec du papier abrasif avant chaque essai. La DCO, la décoloration, le pH, les polyphénols sont mesurés après chaque traitement pour déterminer les conditions opératoires optimales.

\section{Résultats et discussion}

\section{I Caractéristiques des échantillons}

Lors de cette étude, les essais ont été réalisés sur les eaux résiduaires diluées à $10 \%$. Ces eaux usées ont été choisies à cause de leur concentration en matière organique relativement élevée. Le tableau I ci-dessous présente les caractéristiques des margines utilisées.

\begin{tabular}{|c|c|c|}
\hline \multirow{3}{*}{$\begin{array}{l}\text { Tableau } 1 \text { : } \\
\text { Caractéristiques } \\
\text { des margines étu- } \\
\text { diées. }\end{array}$} & Paramètres & $\begin{array}{c}\text { Résultats obtenus sur } \\
\text { margine brute }\end{array}$ \\
\hline & PH & 4,4 \\
\hline & $\operatorname{DCO}(g / L)$ & 152,10 \\
\hline \multirow{6}{*}{$\begin{array}{l}\text { L'effluent est } \\
\text { acide et fortement } \\
\text { chargé } \\
\text { (DCO = I52 g/L, } \\
\text { concentration en } \\
\text { polyphénols = } 15\end{array}$} & $\operatorname{MES}(g / L)$ & 11,5 \\
\hline & Conductivité & 8,3 \\
\hline & $(\mathrm{ms} / \mathrm{cm})$ & 15 \\
\hline & Phénols (g/l) & marron \\
\hline & Couleur & \\
\hline & & \\
\hline \multicolumn{3}{|c|}{$\begin{array}{l}\text { La coloration est considérée comme un indicateur de la pol- } \\
\text { lution organique dissoute [12]. Les échantillons de margines } \\
\text { étudiés présentent une coloration sombre (marron). Cette } \\
\text { coloration est due en grande partie à la présence des com- } \\
\text { posés phénoliques [I8] et sa variation est liée à la distribution } \\
\text { des types de ces composés [19]. Par ailleurs, les analyses par } \\
\text { spectroscopie UV visible (figure I) montrent l'existence des } \\
\text { bandes d'absorbance UV aux alentours de } 278 \text { nm qui cor- } \\
\text { respondent au domaine d'absorption des polyphénols. }\end{array}$} \\
\hline
\end{tabular}


Figure 1 : Absorbance UV visible des margines diluées.

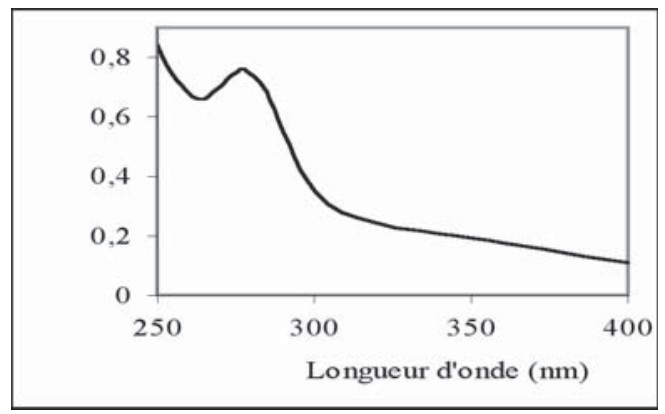

\subsection{Paramètres influençant le traitement des margines}

\subsection{Influence du pH sur l'électrocoagulation}

Le $\mathrm{pH}$ initial de la margine est un facteur opératoire important qui influence les performances du processus électrochimique. Pour démontrer son effet sur le rendement du traitement, le pH de la margine étudiée est ajusté entre 2 et I0. Le temps de traitement électrochimique et le potentiel sont fixés à 7 minutes et $22 \mathrm{~V}[17]$.

Les résultats obtenus sont représentés sur la figure 2, où l'on remarque qu'il y a deux parties distinctes :

Figure 2 : pH après le traitement électrochimique en fonction du pH initial.

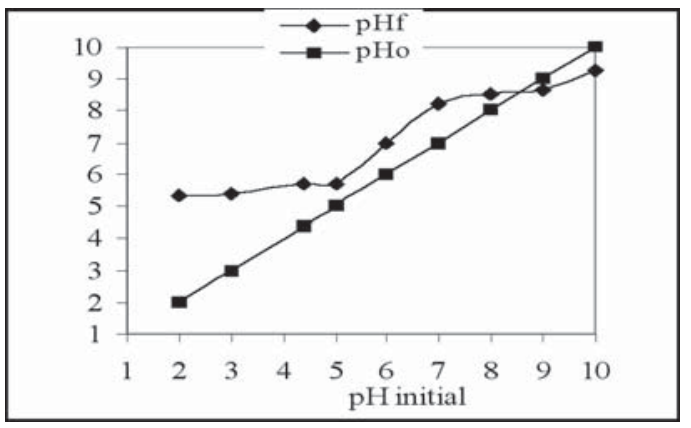

- Pour des $\mathrm{pH}$ initiaux acides ( $\mathrm{pH}=2$ à 7), le pH augmente pendant le traitement. Ceci est dû essentiellement à la réduction des ions $\mathrm{H}^{+}$au niveau de la cathode avec un dégagement d'hydrogène (qui est observé) et une production d'ions $\mathrm{Fe}^{+3}$ suivant les réactions :

$$
\begin{gathered}
2 \mathrm{H}^{+}+2 \mathrm{e}^{-} \rightarrow \mathrm{H}_{2} \\
\mathrm{Fe}+3 \mathrm{H}^{+} \rightarrow \mathrm{Fe}^{+3}+3 \mathrm{e}^{-}
\end{gathered}
$$

- Pour les $\mathrm{pH}$ basiques ( $\mathrm{pH} \geq 9$ ), il y a une faible diminution de $\mathrm{pH}$ due à la consommation des ions $\mathrm{OH}^{-}$selon la réaction suivante :

$$
\mathrm{Fe}^{3+}+3 \mathrm{OH}^{-} \rightarrow \mathrm{Fe}(\mathrm{OH})_{3}
$$

L'influence du pH sur la réduction de la DCO, de la coloration et des polyphénols a été étudiée. La variation de la DCO est représentée sur la figure 3. Sur toute la gamme de $\mathrm{pH}$, les valeurs finales de DCO obtenues sont de l'ordre de $10 \%$ (environ $15 \%$ pour les $\mathrm{pH}$ très acides). Le pourcentage d'élimination de la $\mathrm{DCO}$ accuse 50,7 \% à pH $=7$ de la margine traitée.

Figure 3 : Variation de la quantité de la DCO de la margine traitée en fonction du $\mathrm{pH}$.

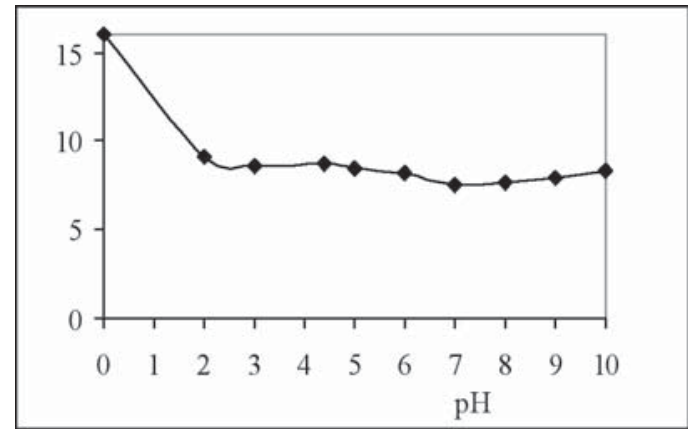

Figure 4 : Absorbance UV visible pour différentes valeurs de pH.

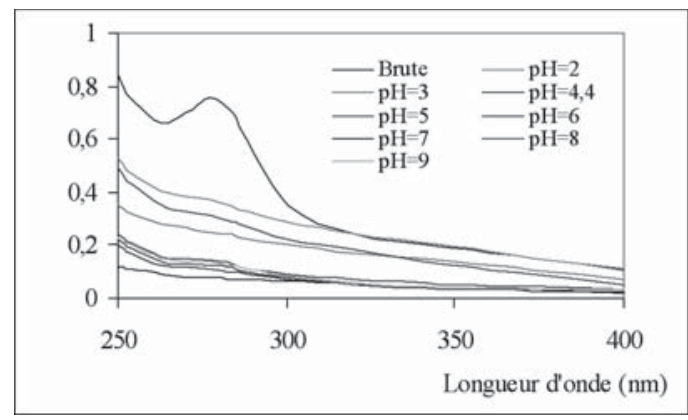

D'après la figure 4, on constate que le changement d'allure des spectres UV/visible est caractérisé par une diminution de l'intensité d'absorption à $278 \mathrm{~nm}$ en fonction du $\mathrm{pH}$, ce qui correspond à la diminution de la matière organique et particulièrement des polyphénols.

La mesure de l'absorbance, indice de la coloration du rejet à $395 \mathrm{~nm}$ (figure 5), montre que le pourcentage de la décoloration atteint $80 \%$ pour les $\mathrm{pH}$ supérieurs à 2 et atteint $97,8 \%$ pour les $\mathrm{pH}$ supérieurs à 7 . On note également une réduction de $50 \%$ de la DCO.

Figure 5 : Pourcentage d'élimination de la DCO, absorbances à $395 \mathrm{~nm}$ et à $278 \mathrm{~nm}$ de la margine traitée en fonction du $\mathrm{pH}$.

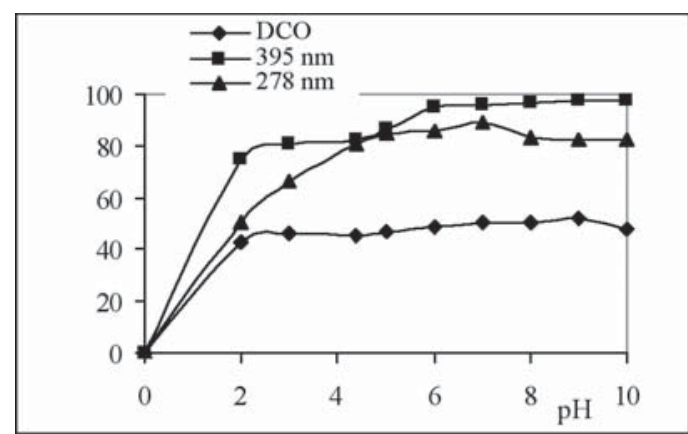




\subsubsection{Cinétique du traitement électrochimique}

La détermination du temps de traitement électrochimique est nécessaire afin de connaître les conditions expérimentales optimales pour la dégradation de la matière organique nuisible (DCO et polyphénols) et la décoloration de l'effluent de margine. Pour faire cette étude cinétique, nous avons traité les échantillons à $\mathrm{pH}=7$ avec un courant imposé de $0,5 \mathrm{~A}$ et un potentiel de $22 \mathrm{~V}$ [6].

Les figures 6 et 7, qui représentent respectivement l'évolution de la DCO et les absorbances UV à $278 \mathrm{~nm}$ et à $395 \mathrm{~nm}$, montrent qu'on a une forte diminution de la DCO qui atteint presque la moitié après 4 minutes et qui devient stable après I 5 minutes de traitement avec une concentration finale de $4,2 \mathrm{~g} / \mathrm{L}$.

Figure 6 : Variation de la quantité de la DCO de la margine traitée par électrocoagulation en fonction du temps.

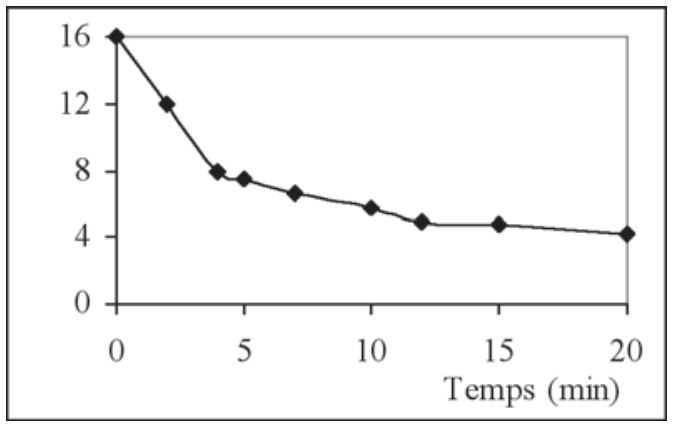

Dans la figure 7, on remarque que l'absorbance UV à 395 nm a fortement diminué après 20 minutes d'électrolyse et que l'absorbance à $278 \mathrm{~nm}$ devient presque stable à elle-même après 12 minutes de traitement.

Figure 7 : Absorbance UV maximals de la coloration à $395 \mathrm{~nm}$ et des phénols à $278 \mathrm{~nm}$ en fonction du temps.

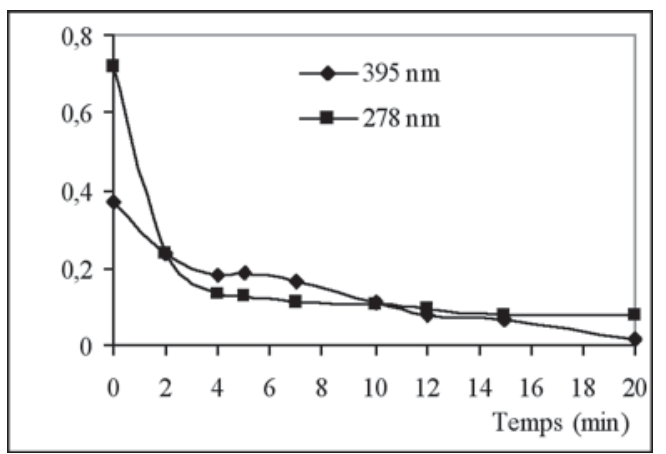

La figure 8, représentant les pourcentages d'élimination de la $\mathrm{DCO}$, la décoloration et l'absorbance UV à $278 \mathrm{~nm}$, montre que le pourcentage de décoloration atteint $95 \%$ au bout de 20 minutes alors que celui d'élimination de l'absorbance UV à $278 \mathrm{~nm}$ accuse $89 \%$ après 15 minutes de traitement. La réduction de la DCO est de $70 \%$. D'où la faisabilité de notre méthode de traitement pour l'élimination des molécules non biodégradables ainsi la décoloration presque totale des effluents.
Figure 8 : Pourcentage d'abattement de la DCO, des absorbances à $278 \mathrm{~nm}$ et à $395 \mathrm{~nm}$ en fonction du temps.

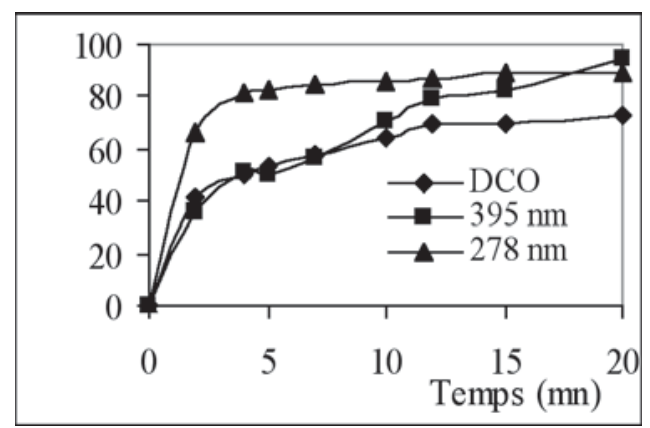

D'après la figure 9, on observe que l'élimination de la DCO est accompagnée par la formation de matières solides en suspension (MES). On note qu'on a une masse qui continue à augmenter jusqu'à 15 minutes. Ceci peut être dû à l'élimination des ions $\mathrm{Fe}^{3+}$ solubles provenant de l'oxydation des électrodes métalliques qui permettent d'assurer le phénomène de coagulation et par conséquent la formation des boues [7].

Figure 9 : Masse de filtrat en fonction du temps de traitement électrochimique.

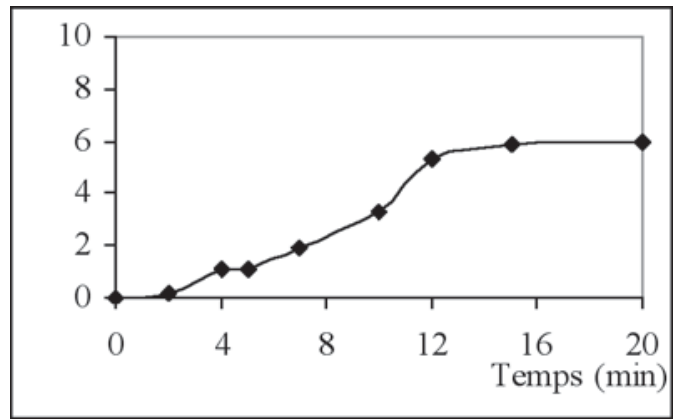

\subsubsection{Influence du courant imposé}

L'étude de la décoloration et de l'élimination de la matière organique par la méthode électrochimique nécessitent l'optimisation de la densité du courant à imposer lors de l'électrolyse afin de minimiser les pertes énergétiques. Une étude a été effectuée $(\mathrm{pH}=7)$ pendant un temps d'électrolyse de 15 minutes pour des margines diluées à $10 \%$.

La figure 10 , qui présente le $\mathrm{pH}$ final en fonction du courant imposé, montre que la variation du pH final par rapport au $\mathrm{pH}$ initial est importante.

Figure 10 : Variation du pH final en fonction du courant imposé.

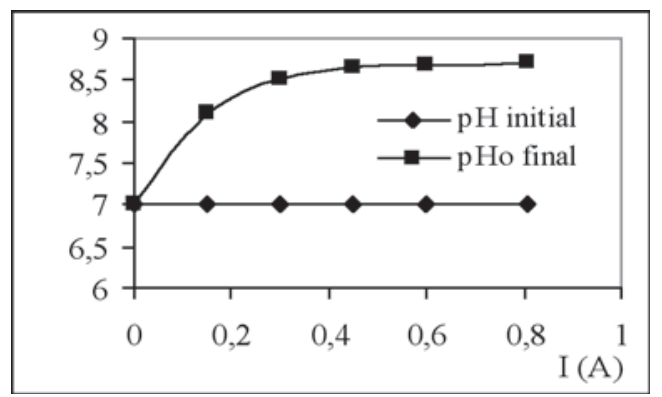


La figure I | montre que la décoloration est totale. Ainsi, l'abattement de la matière organique en composés phénoliques (qui absorbent à $278 \mathrm{~nm}$ ) est très important. L'augmentation du $\mathrm{pH}$ après le traitement électrochimique peut être expliquée par la production des ions $\mathrm{OH}^{-}$par électrolyse de l'eau avec dégagement de l'hydrogène sur la cathode selon la réaction suivante :

$$
2 \mathrm{H}_{2} \mathrm{O}+2 \mathrm{e}^{-} \rightarrow 2 \mathrm{OH}^{-}+\mathrm{H}_{2}
$$

La variation de l'absorbance UV à max $=395$ nm dépend fortement du courant imposé pendant l'électrolyse (figure I I). Sur cette même figure, on observe que pour $1 \leq 0,5 \mathrm{~A}$, la décoloration et l'élimination de la bande d'absorption UV à $=278 \mathrm{~nm}$ est importante (ce qui explique que le potentiel d'oxydation du fer en ions $\mathrm{Fe}^{3+}$ est atteint) et que pour | > 0,5 A, la décoloration est presque totale.

Figure 11 : Absorption UV des phénols à $278 \mathrm{~nm}$ et du colorant à $395 \mathrm{~nm}$ en fonction du courant imposé.

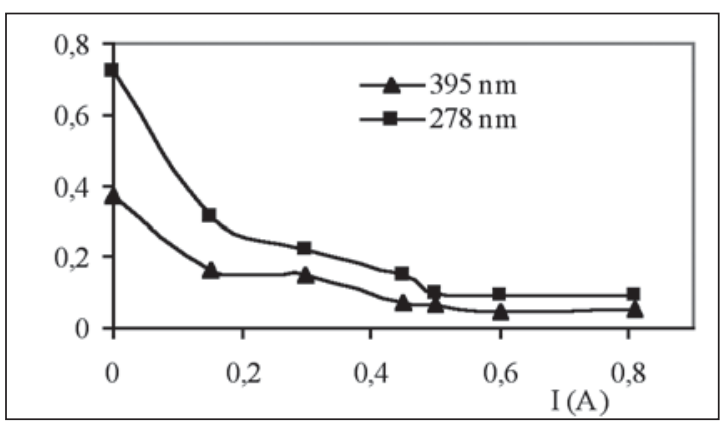

La figure 12, qui présente la variation de la DCO en fonction du courant imposé, montre que la matière organique diminue au fur et à mesure que le courant imposé augmente et atteint un palier quand on impose des courants supérieurs à 0,5 A.

Figure 12 : Variation de la DCO en fonction du courant d'électrolyse.

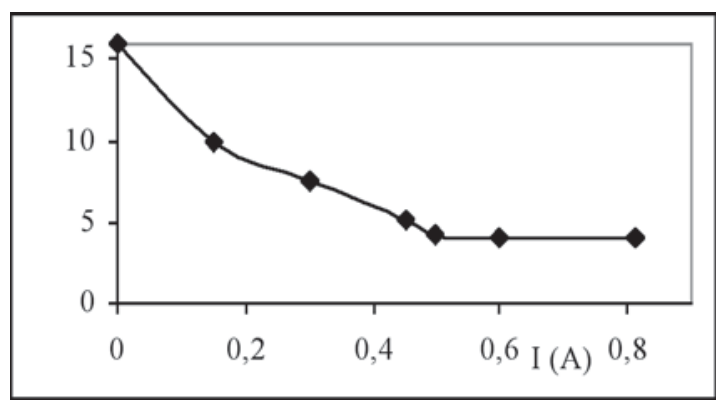

La représentation de ces résultats sous forme des pourcentages d'élimination est illustrée dans la figure 13.

II est clair que la décoloration du rejet de la margine atteint $95 \%$ pour les courants d'électrolyse ou I $\geq 0,5 \mathrm{~A}$. De même, la variation du pourcentage d'élimination de l'absorption à $278 \mathrm{~nm}$ accuse environ $89 \%$ à partir de I $\geq 0,5 \mathrm{~A}$. En ce qui concerne la DCO, la figure 13 montre que l'abattement de la matière organique qui existe dans l'eau usée des huileries d'olives devient de plus en plus important en augmentant le courant imposé.
Figure 13 : Pourcentage d'élimination de la DCO des absorptions à $278 \mathrm{~nm}$ et à $395 \mathrm{~nm}$ en fonction du courant d'électrolyse.

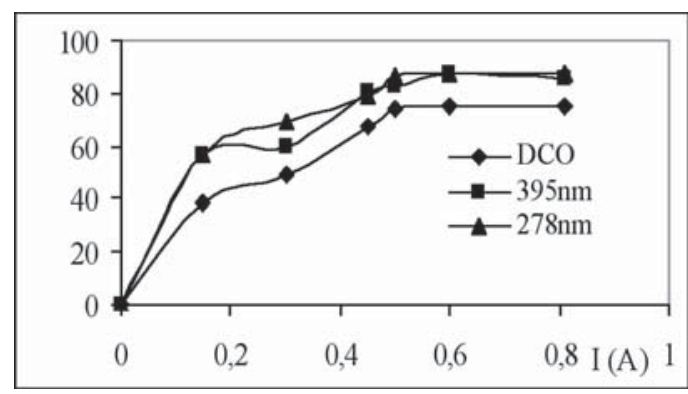

Cet abattement dépasse 75 \% pour des courants d'électrolyses | > $0.5 \mathrm{~A}$.

\section{Conclusion}

Une étude du procédé d'électrocoagulation d'une margine provenant d'une unité de production d'huile d'olive a été réalisée. Trois facteurs (le $\mathrm{pH}$ initial, le temps d'électrolyse et le courant imposé) influencent l'efficacité du traitement. Les valeurs optimales de $\mathrm{pH}$, du temps d'électrolyse et du courant imposé ont été évaluées respectivement à 7 unités de $\mathrm{pH}, \mathrm{I} 5$ minutes de traitement et 0,5 A.

Dans ces conditions, nous avons obtenu une décoloration de $95 \%$, une réduction des phénols qui dépasse $89 \%$ et un abattement de la DCO de $75 \%$.

\section{Références bibliographiques}

[I] M. Mouncif, M. Faid, A. Achkari-begdouri, R. Lhadi, A. Biotechnological, Valorization and treatment of olive mill waste waters by selected yeast strains ; Grasas y Aceites, vol. 46Fasc., 1 995, 344-348.

[2] C. Paredes, J. Cegarra, A. Roig, M.A. Sanchez Monedero, M.P. Bernal, Characterization of olive mill wastewater (alpechin) and its sludge for agricultural purposes, Bioressource Technologie 67, 1999, p : | | | - | | 5.

[3] M. Kissi, Thèse de doctorat, 2001 ; Faculté des Sciences, El Jadida, Maroc.

[4] L. Lesage-Meessen, D. Navarro, S. Maunier, J-C. Sigoillot, J. Lorquin, M. Delattre, J-L. Simon, M. Asther, M. Labat, Simple phenolic content in olive oil residues as a function of extraction systems, Food Chemistry 75 (200 I) 50 I-507, pp. I0I31020.

[5] M. I. Aguilar, J. Saez, M. Lloréns, A. Soler, J. F. Ortuno, Nutrient removal and sludge production in the coagulationflocculation process, Water research 36, 2002, p. 2910-2919.

[6] T.H. Ergüder, E. Güven, G.N. Demirer, Anaerobic treatment of olive mill wastes in batch reactors; Process Biochemistry, Vol. 36 , 2000, pp. 243-248. 
[7] Ouafi Oukili, Mehdi Chaouch, Mohamed Rafiq, Mohammadi Hadji, Moktar Hamdi, Mohamed Benlemlih, Bleaching of olive mill wastewater by clay in the presence of hydrogen peroxide, Ann. Chim. Sci. Mat, 200I, 26 (2), pp. 45-53.

[8] Edoardo Turano, Stefano Curcio, Maria G. De Paola, Vincenza Calabrò, Gabriele lorio, An integrated centrifugation-ultrafiltration system in the treatment of olive mill wastewater, Journal of Membrane Science, 2002, pp. 519-531.

[9] F.Flouri, D. Sotirchos, S. loannidou \& C. Balis ; Decolorization of olive oil mill liquid wastes by chemical and biological means; International Biodeterioration \& Biodegradation (1996) 189-192.

[10] R. Borja, R. Martin, V. Alonso, I. Garcia, C.G. Banks, Influence of different aerobic pretreatments on the kinetiks of anaerobic digestion of olive mill wastewater, Wat. Res. Vol. 29 , $n^{\circ} 2$, 1995, pp : 489-495.

[ I I] Sayadi S., Zogani F., Ellouz R. Decolorization of olive mill waste waters by free and immobilized Phanerochaete chrysosporium cultures.Appl. Biochem. Biotechnol.(1996) 56 : 265-276.

[12] Vinciguerra, V., D'Annibale A., Delle Monache G., Sermanni. G. G. Correlated effects during the bioconversion of waste olive waters by Lentinus Edodes. Bioresource Technol. (1995) 51: 221-226.

[I 3] A. Jarmany, A. Kheribech, M. Mountadar ; La décoloration des rejets liquides de textile par électrocoagulation ; Phy. Chem. News 6 (2002), I 01 - 109.

[14] A. B. Paul, India, Electrolytic treatment of turbid water in package plant ; F Water Quality and Supply ; New Delhi, India, 1996, pp. 286-288.

[15] G. Saracco, L. Solarino, R. Aigotti, V. Specchia, M. Maja ; Electrochemical oxydation of organic pollutants at low electrolyte concentrations ; Electrochemica Acta 46 (2000), 373-380.

[ I 6] B Idbelkas, D. Takky ; Traitement électrochimique d'eaux usées chargées de phénol : Etude comparative sur des électrodes de dioxyde de plomb et de platine ; Ann. Sci. Mat, 200।, 26(2), pp. 33-44.

[17] W. Phutdhawong, S. Choowwanapoonpohn, Buddhasukh; Electrocoagulation and subsequent recovery of phenolic compounds; Analytical Sciences, october 2000, Vol. 16, p. 1083.

[18] Gonzales, M. D., Moreno E., Quevedo-Sarmiento J., Ramos-Cormenzana A. Studies on antibacterial activity of waste waters from olive oil mills (alpechin): Inhibitory activity of phenolic and fatty acids. Chemosphere. (1990) 20 :423-432.

[19] Hamdi, M., Khadir A., Garcia J. L. The use of Aspergillus niger for the bioconversion of olive mill wastewaters. Appl. Microbiol. Biotechnol. (199I) 34 : 828-831. 